\title{
Survey of digital radio standards and updating for rollout in Brazil
}

Ramon Mayor Martins

Elton Ferreira Broering

Danilo Bedaque

Jean Michel de Souza Sant'ana

Thiago Henrique Bonotto da Silva 


\title{
Survey of digital radio standards and updating for rollout in Brazil
}

\author{
Ramon Mayor Martins, Elton Ferreira Broering, Danilo Bedaque, Jean Michel de Souza Sant'ana, \\ Thiago Henrique Bonotto da Silva
}

\begin{abstract}
Currently, the spectrum resource is sparse, requiring a system that optimizes use this. An option for radio broadcast is the use of digital radio techniques. These techniques are available standards DAB, DRM, IBOC and have extensive advantage over analog systems, for example, flexibility and audio quality. The choice of the most appropriate digital radio technique is a very important problem, and check of multilateral performances is necessary to countries considering the release of digital radio broadcasting service. In Brazil some tests with digital radio were performed, but no further definition of what standard to use.

This paper aims establish some technical comparison of the leading digital radio standards available. Another goal is to present the movements of activities with digital radio in Brazil. Performing some direct comparisons between the patterns, it was observed that DRM is interesting in order to coverage and being open source, while IBOC (HD Radio) is a hybrid system, however proprietary.
\end{abstract}

Index Terms-Broadcast, Digital Radio, Engineering, Telecommunications.

\section{INTRODUCTION}

$\mathrm{B}$ razil has experienced some discussions and optimization strategies of the electromagnetic spectrum resources and improvement of the telecommunications system. The most current strategies correspond as follows: migration of AM radio stations to FM in decree of November 7, 2013 signed by President Dilma Rousseff, which enables higher audio quality and robustness to interference stations that currently operate in AM; the incorporation of the channels 5 and 6 of analog TV to accommodate the FM bands, which became known as extended FM; the migration from analogue TV to digital TV, which according to the Ministry of Communications has changed the analog TV shutdown schedule for 2018. In this context, another strategy was taken in 2014 where Anatel (National Agency of Telecommunications) published notice of auction of the 700 MHz band, which focused channels (52-69) TV UHF to meet the demands of $4 \mathrm{G}$ telephony, rearranging the UHF channels 14 to 51 .

Adequacy of these initiatives, it also highlights the discussion on the introduction of digital technology in radio broadcasting system in Brazil. The digital radio standards has

Paper submitted in June 30, 2016. This work was supported in part by the Federal Institute of Santa Catarina (IFSC), campus São José.

Ramon Mayor Martins is with the IFSC, Telecommunications Department, Brazil (e-mail: ramon.mayor@ifsc.edu.br).

Elton Ferreira Broering is with the IFSC, Telecommunications Engineering, Brazil (e-mail: eltonbroering@gmail.com). extensive advantage over analog systems. Among some of these include: audio quality, service reliability, programming, coverage flexibility receiver, spectrum, and power efficiency, as well as new business opportunities for the broadcaster. Due to inadequate spectrum resources and increasing demands for high-quality multimedia services, traditional analog audio broadcasting is being migrated into digital radio around the world [1].

Recently various digital radio broadcasting techniques and standards have been proposed, and many countries have been considering converting their analog radio broadcasting into digital broadcasting. However, since a small number of countries have achieved the digital conversion of their analog radio broadcasting (some European countries and the U.S.), most countries still face converting their analog radio broadcasting service into digital service [2].

In Brazil, in March 2007, the advisory board of the Digital Radio was created in order to advise the Ministry of Communications in planning the implementation of digital radio in Brazil. The board is composed of representatives of civil society, the federal government, including Anatel and the Ministry of Communications; broadcasting sectors (commercial, educational, community and public); industry (reception, transmission and audiovisual); academic institutions, and advertisers.

In 2010, the Brazilian Digital Radio System (SBRD) was established by Ministry of Communications decree number 290 [3]. According to Anatel, in partnership with performers of different stations broadcasting services, the Ministry of Communications has performed technical tests to check the performance of different models [4].

The choice of the most appropriate digital radio technique is a very important problem, and check of multilateral performances is necessary to countries considering the release of digital radio broadcasting service [2]. This paper aims establish some technical comparison of the leading digital radio standards available. In the section 2 will be presented techniques and their characteristics, they being the DAB (Digital Audio Broadcasting), DRM (Digital Radio Mondiale) and IBOC (HDRadio) and FMeXtra. In section 3, the comparisons between these techniques. In section 4 the implementation perspective in Brazil. Finally, in section 5, the conclusions about the digital radio standards.

Danilo Bedaque is with the IFSC, Telecommunications Engineering, Brazil (e-mail: elton@lamar.colostate.edu)

Jean Michel de Souza Santana is with the IFSC, Telecommunications Engineering, Brazil (e-mail: jeanssant@gmail.com).

Thiago Henrique Bonotto da Silva is with the IFSC, Telecommunications Engineering, Brazil (e-mail: thbonotto@gmail.com). 


\section{SURVEY OF DIGITAL RADIO STANDARDS}

There are currently four types of Digital Radio (DR) systems deployed throughout the world. These systems are known as digital audio broadcasting (DAB) system, digital radio mondiale (DRM), in-band on-channel (IBOC), and satellite digital audio radio. These system offer the following advantages according to [5]. DR reception is largely immune to interference, they provide fixed, mobile, and portable reception using simple low-gain antennas. DR is efficient in the use of the limited radio frequency spectrum and they have the capability of operation from terrestrial and/or satellite transmitters. Besides that, value-added system feature will allow enhancements such as text, graphics, and still-pictures.

These features provide the opportunity to introduce new innovative services, such as multimedia radio-with-pictures and broadcast web sites on commercial amplitudemodulation (AM) and frequency-modulation (FM) receivers.

\section{A. DAB (Digital Audio Broadcast)}

The DAB (Digital Audio Broadcast) family includes DAB and $\mathrm{DAB}+$ for digital radio and $\mathrm{DMB}$ (Digital Multimedia Broadcasting) for mobile TV.

The original DAB started its development in 1981 on Institut für Rundfunktechnik (IRT) and was completed by the EUREKA 147/DAB project. The specification was finalized after some public demonstration on early 90's in 1993. It was adopted by ITU-R as a standard in 1994, the european community in 1995 and by ETSI in 1997. This first standard uses the MPEG Audio Layer II codec.

It was the first standard based on OFDM (Orthogonal Frequency Divison Multiplexing), technique that was not very known in communication systems.

According to [7], DAB is very well suited for mobile reception and provides very high robustness against multipath reception. It allows use of single frequency networks (SFNs) for high frequency efficiency. This technique is also able to transmit personalized data, based on the program, radio station and other service like traffic information.

The frequency bands in DAB according with [7] is band III (174-230 MHz / $240 \mathrm{MHz}$ in some countries). An external antenna is needed for a high quality reception however the band provides a wide range of coverage and provides high penetration on buildings. Band L (1452-1479.5 MHz) is used in some countries where Band III is not available or as a backup band. It does not need an external antenna, what is an advantage for mobile phones. Band $\mathrm{L}$ is used on urban areas with high buildings on both side of streets due to a reflective characteristic of this band. It allows a good reception without line of sight, but the reception at indoor ambient is limited. Even so, this band can be useful for some applications like local information distribution systems inside buildings, e.g. in department store, railway stations.

The benefits of DAB, according with [6] is: quality of service, superior sound quality (close to CD quality), perfect reception condictions; wide range of value-added services, in other words, information services (news headline, urgent information); universal system layout, high level of standardization of basic principles and transmission tools, unique system design (services available on terrestrial, cable and satellite networks providing national, regional, local and international coverage), wide choice receivers (it possible to access $\mathrm{DAB}$ services from fixed equipment, mobile and portable radio); flexibility of multiplex configuration (the arrangement of services may be changed to match the need of the providers of programs or data services, without interrupting ongoing services), bit rate flexibility (the programmer provider can choose the bit rate for an audio program according to its quality, for instance less than 100 $\mathrm{kbit} / \mathrm{s}$ for a pure speech, $128 \mathrm{kbit} / \mathrm{s}$ for mono and $256 \mathrm{kbit} / \mathrm{s}$ for stereo music); transmission efficiency, lower transmission costs for broadcasters and transmitter (DAB needs only a fraction of the electrical energy compared to conventional $\mathrm{AM}$ and FM transmitter).

The future of DAB is the DAB + development, even with the MPEG Audio Layer III (MP3) popularization in digital music player market, the standard was overcome in efficiency (same audio quality with lower bit rate) and performance by MPEG-4 (HE AAC v2 codec). Because of this, there was an integration of AAC (Advanced Audio Coding) with DAB and in 2007 February, DAB + was developed. The physical layer remained the same of $\mathrm{DAB}$, with only a few new applications, transport protocols and a second error correction layer. Therefore it is possible an structure able to multiplex both technologies [8]. DAB + uses a Reed Solomon coding, a more sophisticated technique than the Viterbi encoder used by DAB. This guarantee a better bit error ratio in the reception [2]. Recently, a new audio codec has been developed: the USAC (Unified Speech and Audio Coding), and it has been implemented in new DAB + systems. "To maximize the performance of USAC audio codec, variable bit rate (VBR) delivery scheme instead of constant bit rate (CBR) scheme used in $\mathrm{DAB}+$ is designed and USAC decoder is inserted to the receiver" [9].

Many countries, mainly in Europe, eastern Asia and Oceania, have DAB regularized or are interested on implementing this systems. Norway, Switzerland and the United Kingdom have almost $100 \%$ of DAB coverage. Others like Turkey, China and South Africa are on trial and some are interested like Russia and Ukraine [10].

\section{B. $D R M$ (Digital Radio Mondiale)}

DRM is a digital radio standard developed and managed by an open international consortium. Therefore it is possible to develop free software for adoption and customization of default. Businesses and universities can create, optimize and deploy it in various electronic devices. The DRM works with high quality both in the AM medium and shortwave using DRM30 mode, as the FM/VHF band, using the DRM+ [11].

The International Telecommunications Union (ITU) recognized the DRM to broadcasting bands below $30 \mathrm{MHz}$ in the year of 2002 by the recommendation BS1514-1. The European Telecommunications Standards Institute (ETSI) also recognized the DRM like a digital sound broadcasting system which can be used as a replacement of the analogue AM sound broadcasting services. Finally in 2003, the International Electrotechnical Committee (IEC) approves the DRM like International Standard [12].

The DRM system uses COFDM (Coded Orthogonal Frequency Division Multiplex). Then all analog data is digitized and divided into several slightly spaced subcarriers within the broadcast channel. The parameter coding and OFDM can be altered to allow the DRM function in different environments with different propagation characteristics. With the right choice of parameters you can adjust the transmission with the best combination of power, robustness and 
transmission rate. The modulations used in DRM are 4QAM, 16QAM and 64QAM depending on the transmission mode. There are five transmission modes, according to [11], as shown in the Table 1.

TABLE I

TRANSMISSION MODES USED IN DRM

\begin{tabular}{|c|c|c|c|c|}
\hline Mode & $\begin{array}{c}\text { MSC QAM } \\
\text { options }\end{array}$ & $\begin{array}{l}\text { Bandwidth } \\
\text { options (kHz) }\end{array}$ & Typical uses & \\
\hline A & 16,64 & $4.5,5,9,10,18,20$ & LF \& MF ground-wave, $26 \mathrm{MHz}$ band line-of-sight & \multirow{4}{*}{$\begin{array}{l}\text { DRM30 } \\
\text { modes }\end{array}$} \\
\hline B & 16,64 & $4.5,5,9,10,18,20$ & HF \& MF transmission on sky-wave & \\
\hline C & 16,64 & 10,20 & Difficult sky-wave channels on HF & \\
\hline D & 16,64 & 10,20 & NVIS sky-wave (highest Doppler \& delay spread) & \\
\hline $\mathrm{E}$ & 4,16 & 100 & VHF transmissions in the bands above $30 \mathrm{MHz}$ & DRM+ \\
\hline
\end{tabular}

The A mode is used for a higher transmission rate using ground-waves and line-of-sight. The B-mode is generally used forsky-wave. The $\mathrm{C}$ and $\mathrm{D}$ modes are used when propagation conditions are more severe like in long distances with multiple hops or when very strong reflections occur. The E mode is used in the VHF band.

The DRM can use the Meltzer-Moser MPEG-4 HE AAC v2 (ISO / IEC), but with a greater robustness against fading channel the HE-AAC v2 is the most widely used standard. DRM system is formed by the service description channel (SDC) which carries the information for decoding the main service channel (MSC), the fast-access channel (FAC) containing the OFDM signal properties and the SDC and MSC configuration and the MSC itself, which encodes the frame generated by the multiplexer [13]. Some of the most important feature of the DRM are the possibility of reuse of the radio spectrum, CD quality sound, terrestrial based DR, wireless data services, backward compatibility with analog radio and interference immunity.[5]

Currently the main radio stations operating with DRM are Vatican Radio, Radio Exterior of Spain, Radio New Zealand International and BBC Radio in England.

\section{IBOC (HDRadio)}

IBOC (in-band on-channel) refers to a method of transmitting DR broadcast signals centered on the same frequency as an existing AM or FM station (referred to as AM and FM systems) but occupying the sidebands above and below the station's center frequency. IBOC is a hybrid method, transmitting digital and analog radio on the same frequency. A major IBOC system is called HDRadio, proprietary of Ibiquity.

IBOC systems are comprised of four building blocks according to [5]: audio source coding, channel coding, modulation/demodulation techniques and blending.

The first is audio source coding, that means: an audio codec is a source encoding device that filters out those parts of an analog signal that are irrelevant to the human ear. When decoded, the signal will not be identical to the original but will be perceived to be the same.

The second, output stream from the audio codec is encoded using forward error correction (FEC) and interleaving in the transmission system. This greatly improves the reliability of the transmitted information by carefully adding redundant information used to correct errors occurring in the transmission path. Advanced FEC coding techniques have been specifically designed for AM systems based on detailed interference studies to exploit the non-uniform nature of interference in the AM bands.

The third block, modulation/demodulation techniques: a modem is a device that modulates a signal or demodulates it. AM systems use quadrature AM scheme in conjunction with orthogonal frequency division multiplexing (OFDM). FM systems also uses OFDM modulation but with the carriers modulated with quadrature phase-shift keying (QPSK) modulation scheme.

The last block is blending: this is a technique employed in IBOC DAB systems to seamlessly switch between digital-toanalog signals. Essentially, blending allows transition from the instantly acquired analog signal (such as when the receiver is first turned on) to the digital signal (after the receiver has acquired, decoded, and processed the signal). Once the digital signal is acquired, the receiver will transition to it in a seamless fashion. Should the digital signal become corrupted the receiver will seamlessly switch to the analog signal.

The typical coherent bandwidth in wide area applications is more than $1 \mathrm{MHz}$. Therefore, even with aggregation, the IBOC is a relatively narrowband system and does not possess sufficient frequency diversity in a fading environment. As a result, its performance is particularly poor for stationary and low-mobility receivers where limited time domain diversity can be explored. In order to improve the performance, a frequency hopping option is adopted to introduce frequency diversity into the system [1].

IBOC uses LDPC codes, that is widely adopted in nowadays wireless communication standards for their Shannon-limit approaching performance and their inherent parallel structure for fast decoding [1].

Currently, several radio stations in the United States use the HD Radio.

\section{FMeXtra (VuCast)}

FMeXtra was an in-band on-channel system created by Digital Radio Express now marked as VuCast. This system uses the remaining capacity with FM radio frequency spectrum. Usually each frequency is a portion from 0 to 59 $\mathrm{kHz}$ for audio, the stereo pilot carrier and the Radio Data System (RDS) [14].

FMeXtra makes use of free present spectrum portion allowing that the digital signal is transmitted in addition to the analog signal, like SCA, between $53 \mathrm{kHz}$ and $99 \mathrm{kHz}$.

FMeXtra technology can deliver $128 \mathrm{k}$ bits/second in stereo configurations. According to [18], the most powerful realization of FMeXtra occurs when the analog broadcast is mono audio as shown below. In this configuration, FMeXtra can use all the bandwidth between $20 \mathrm{kHz}$ and $99 \mathrm{kHz}$, and can deliver over $256 \mathrm{kbits} / \mathrm{sec}$, which is more bits than a dedicated ISDN data line.

By using AAC+ codec in combination with MPEG 4, the sound quality is greatly improved. The quality moreover it depends on the number of stations that digitally broadcast on the same frequency. The more stations, becomes all the less the sound quality [14].

Currently, some stations in China and United States using FMeXtra (VuCast).

\section{COMPARISON BETWEEN THE DIGITAL RADIO STANDARDS}

Some factors can serve as a comparison between the digital radio standards available in the market such as radio spectrum 
reuse, specific allocation of radio spectrum for digital radio, audio quality, multimedia features, compatibility with the analog system, immunity to interference, power requirement, coverage and cost of deployment.

The radio spectrum reuse: DAB is the only system that does not reuse. DRM is able to transmit up to four programs at the same frequency. The DRM occupies the same bandwidth of an analog signal. HD Radio does the reuse, but uses 2 digital side carriers, as well as analog, occupying the range of 3 channels.

The specific radio spectrum allocation: DAB requires a specific range for your signal.

In the audio quality: all patterns have higher quality than FM stereo. In HD Radio, being a hybrid standard, performs FM broadcasts with CD quality and AM broadcasts with the quality of a stereo FM. About multimedia features: all patterns have this feature. About compatibility with the analog system: the DAB is not compatible, all others are.

Concerning a immunity to interference: all patterns are immune to interference, as are digital standards. As to power requirement: The DRM works with low power, the HD Radio does not work. In terms of coverage: the DRM works on shortwave with DRM30 (AM), the HD Radio does not work on shortwave. The DRM allows different transmission modes can function MF, HF and VHF, and may be configured so as to adapt the transmission according to the need, HDRadio does not use HF.

About the modulation scheme: DRM uses COFDM and QAM modulation according to the mode of transmission and to improve the requisites of the power and transmission rate. The use AM HD Radio systems QAM modulation scheme and the OFDM, since the FM and QPSK systems use OFDM modulation schemes. Both DRM and DAB uses MPEG-4 codec HE AAC v2 codec, to give greater robustness against fading in the channel. Other comparisons can be made, like technology available: DRM is an open, standards and publicly available standards. The HD Radio is a proprietary system with closed technology.

The IBOC systems to seamlessly switch between digitalto-analog signals. Essentially, blending Allows transition from the instantly acquired analog signal (such as When the receiver is first turned on) to the digital signal (after the receiver has acquired, decoded, and the processed signal).

Finally, the difference between HD Radio and FMeXtra is mainly the techniques. FMeXtra, makes use of the subcarrier of an FM signal, while HD Radio makes use of the side band. This allows the signal is not transmitted exactly on the FM frequency of the station, but actually next to the signal. Another big difference is that FMeXtra much cheaper than HD Radio. In addition, HDRadio stations also requires royalties additional pay for the mere use of the system. This involves an amount of thousands of dollars per year. The coverage is similar to FM Stereo, but higher ERP is required in larger urban areas, as with normal FM transmissions [14]. Some comparisons are shown in Table 2.

TABLE II

COMPARISON BETWEEN THE RADIO STANDARDS

\begin{tabular}{|c|c|c|c|c|}
\hline & DAB & DRM & $\begin{array}{c}\text { IBOC } \\
\text { (HDRADIO } \\
\text { ) }\end{array}$ & $\begin{array}{c}\text { FMEXTR } \\
\text { A } \\
\text { (VUCAST } \\
\text { ) }\end{array}$ \\
\hline Technique & $\begin{array}{c}\text { OFD } \\
\text { M }\end{array}$ & $\begin{array}{l}\text { COFDM/4- } \\
16-64 \text { QAM }\end{array}$ & $\begin{array}{c}\text { OFDM/ } \\
\text { QPSK }\end{array}$ & $\begin{array}{c}\text { FM } \\
\text { Stereo } \\
\text { with SCA } \\
\text { portion }\end{array}$ \\
\hline
\end{tabular}

\begin{tabular}{|c|c|c|c|c|}
\hline $\begin{array}{c}\text { Radio } \\
\text { Spectrum } \\
\text { Reuse }\end{array}$ & no & yes & yes & yes \\
\hline $\begin{array}{c}\text { Specific } \\
\text { Allocation of } \\
\text { Radio } \\
\text { Spectrum }\end{array}$ & yes & no & no & no \\
\hline $\begin{array}{c}\text { Audio } \\
\text { Quality }\end{array}$ & yes & yes & yes & yes \\
\hline $\begin{array}{c}\text { Multimedia } \\
\text { Features }\end{array}$ & yes & yes & yes & yes \\
\hline $\begin{array}{c}\text { Compatibilit } \\
\text { y with the } \\
\text { Analog } \\
\text { System }\end{array}$ & no & yes & yes & yes \\
\hline Bands & VHF/ & MF,HF,VH & VHF & VHF \\
& Band & F & & \\
\hline
\end{tabular}

\section{IMPLEMENTATION PERSPECTIVE IN BRAZIL}

Brazil is looking for a flexible standard for scanning radio stations, just as happened with the Digital TV standard choice. The digital radio guidelines in Brazil are given by decree No. 290/2010 of the Ministry of Communications [3]. According to [15], the SBRD (Digital Radio Brazilian System) joined as technical basis signals standards IBOC (HD Radio) standard used in the United States and created by American iBiquity Digital Consortium and DRM (Digital Radio Mondiale), used in Europe. In this respect, the DAB and FMeXtra systems were not listed for testing.

In this paper it was not listed ISDB Japanese standard for digital radio ISDB-Tsb (Integrated Services Digital Broadcasting Terrestrial, Segmented Band). This system was not considered in Brazil to occupy a very broadband, and thus would have to use its own range, which opposite way to spectrum optimization.

In Brazil, digital broadcasts are still in progress, new emissions tests were approved in 2005 and since 2007 tests are conducted using the two signals. Anatel approved 12 stations to perform the tests. Broadcasters who tested the HD Radio were: Sistema Globo de Rádio, Rádio Jovem Pan, RBS, and El Dorado for the regions of São Paulo, Rio de Janeiro, Belo Horizonte, Porto Alegre, Curitiba and Brasília. Broadcasters who tested the DRM were Radiobrás and the Faculty of Technology of Brasília University [15]. The planning of the tests, listed in 2007, with the DRM system in medium waves can be found at [16]. Conforming to [15] by 2014 no decision had been made about the standards. According to the results of the tests none of the standards were satisfactory, the delay in the decision by the two standards did concern among broadcasters grow. In 2013, the chief of staff EBC (Brazilian public broadcaster) stated at the public hearing of the Committee on Science and Technology of the Senate, that no good implementation of digital radio in Brazil if the cost of the equipment is high making limited access.

According to [17], both the AM of the migration process for the FM as well the digital radio deployment process in Brazil is stagnant. Delaying process shutdown of analog TV, decelerate further migration and digital radio has been abandoned by the thought that radio and web radios applications will be the future. In Brazil, the only development of digital radio is supported by $\mathrm{EBC}$, which has been trying for more than a year put a solid and robust DRM shortwave in the Amazon National Radio [17]. 
The latest initiatives in Brazil were testing the $\mathrm{EBC}$ with DRM in the frequency of $9755 \mathrm{kHz}$ in 2015. In 2016, an EBC request to the Ministry of Communications on a definition for Digital Radio Brazilian and the foundation of the ABRADIG (Digital Radio Brazilian Association) with the aim of promoting the implementation of Digital Radio in Brazil.

\section{CONCLUSIONS}

The Digital Radio allows digital audio with higher quality than an analog FM station allows multiprogramming, interactivity, multimedia content, among others. In Brazil there is still the current decree AM migration to FM, which does not provide for the use of digital radio techniques.

For Brazil, the most suitable system is the DRM, it is the only system that meets all frequency bands (MF, HF and VHF), and is an open source system, strengthening the domestic industry in the aspect of development transmitters and receivers. The IBOC (HD Radio) developed and owned by US company Ibiquity is a closed, proprietary system. The audio encoder and protocols for transmission of multimedia content and digital services are also closed.

The system also charges system usage license, broadcasters pay for Ibiquity use rate. HD Radio, being a standard hybrid, keeps with the analog signal, which does not solve the spectrum optimization. Despite the DRM comply with the digital radio guidelines in Brazil given by decree 290/2010 [3], such as social inclusion, cultural diversity, democratization of information, technology transfer for the Brazilian industry, among others, and also provide the efficient use of radio frequency spectrum, with the DRM membership, people will have to buy new appliances. The prices of these new devices may not be accessible.

\section{ACKNOWLEDGMENT}

The authors would like to thank Telecommunications Engineering from the IFSC - Instituto Federal de Santa Catarina, campus São José for their support in the preparation of this work.

\section{REFERENCES}

[1] C. Zhiyong; Liu Huj; X. Shao; X. Guanbin, "System Design for Broadband Digital Radio Broadcasting", IEEE Communications Magazine, vol. 51, no. 4, pp. 58-65, Apr, 2013.

[2] B. Myung-Sun; P. Sora; K. Geon; L. Yong-Hoon; S. Yung-Jeong; I. Chae-Hun; L. Yong-Tae; "Design and Performance Evaluation of Digital Radio Measurement Test Beds for Laboratory Test", IEEE Transactions on Instrumentation and Measurement, vol.62, no. 2, pp. 451-459, Sep, 2012.

[3] Ministério das Comunicações (2010, 03). Portaria no 290, Brasil. [Web]. Available: http://www.mc.gov.br/portarias/25477-portaria-n290-de-marco-de-2010.

[4] Anatel $(2015,03)$ Rádio Digital [Web]. Available: http://www.anatel.gov.br/setorregulado/index.php/radio-digital

[5] B. Dennis, "Digital Audio Around the World", IEEE Vehicular Technology Magazine, vol.5, no. 4, pp. 24-30, Apr, 2010.

[6] H. Wolfgang and L. Thomas, "Digital Audio Broadcasting - Principles and Applications of Digital Radio", $2^{\text {nd }}$ Ed, pp. 2-7, 2013.

[7] World DAB $(2015,08)$. Introduction to Digital Radio Spectrum [Web] Available: http://www.worlddab.org

[8] World $\mathrm{DAB}(2015)$. Introduction to $\mathrm{DAB}$ and $\mathrm{DAB}+[\mathrm{Web}]$. Available: http://www.worlddab.org

[9] B. Myung-Sun; L. Bongho; Y. Kyutae; B. Seungkwon; L. Minsuk; S. Gunsu; K. Ki-Won; L. Hyoungsoo; "Design and Performance Evaluation of Advanced Digital Audio Broadcasting System", MulGraB 7th International Conference on Multimedia, Computer Graphics and Broadcasting, pp. 25-28, Nov. 2015.
[10] World DAB (2015, 08). Global Summary, Digital Radio Broadcasting using DAB family of standards[Web]. Available: http://www.worlddab.org

[11] L. Nigel, "The DRM Digital Broadcasting System - Introduction and Implementation Guide", rev. 2, Sep, 2013.

[12] G. Pietro; I. Pichel; D. Guerra; P. Angueira; J.M. Matias; J.L. Ordiales; A. Arrinda. "Digital Radio Mondiales: broadcasting and reception", IEEE Mediterranean, MELECON Electromechanical Conference, v.2, pp. 485-487, May, 2004.

[13] Heng-Zhou Wu; Wang-Da Chen; Lin-Yu Fu; Xin Zhang. "Study on Digital Radio Mondiale Signal Monitor System for Wireless Communication Security", $9^{\text {th }}$ International Conference CIS Computation Intelligence and Security, pp. 620-623, Dec, 2013.

[14] Radio TV Nederland (2015) Alle Legale AM, FM, DAB en DVB etherzenders in Nederland - Hoe werkt FMeXtra? [Web]. Available: http://radio-tv-nederland.nl/dab/fmextra.html

[15] P. S. Mariana; S. R. Lanna Paula; N. R. S. Brenda, "Rádio Jovem Pan Belém: perspectivas do padrão digital e sua relação com as redes", Intercom - Sociedade Brasileira de Estudos Interdisciplinares da Comunicação, Sep, 2015.

[16] Anatel (2007) Planejamento dos Testes com o Sistema DRM em Ondas Médias em Brasília.

[17] [20] M. Goedert (2014) Brazil - So what now radio? [Web]. Available: http://www.drm.org/?p=4150

[18] VuCast (2016) - FmeXtra [Web]. Available: https://sites.google.com/site/vucastmedia/fmextra

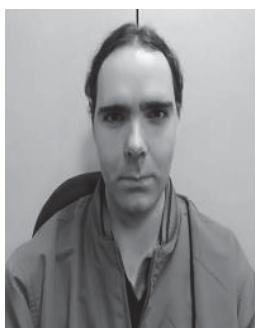

Ramon Mayor Martins was born in Paraisópolis, Minas Gerais in 1982. M.Sc in Telecommunications by the Instituto Nacional de Telecomunicações, PgC Specialist in Telecommunications Systems and B.Eng with emphasis in Telematic. He worked as a researcher at the Inatel Competence Center with embedded system. Currently holds the position of Professor at Telecommunications Engineering in the Instituto Federal de Santa Catarina. He has developed research in areas like: RF systems, antennas project, satellite communications and artificial intelligence.

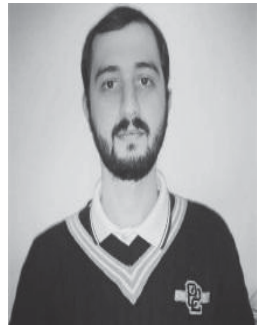

Danilo Bedaque was born in São Paulo, São Paulo, in 1986. Technical majored in telecommunications from Instituto Federal de Santa Catarina in 2006. He is currently pursuing the bachelor's degree in Telecommunications Engineering at the Instituto Federal de Santa Catarina. Currently participate in a research about LoRa technology in Instituto Federal de Santa Catarina. He studies the implementation and performance of the network that will be the first network of this kind in the state and the second in the country.

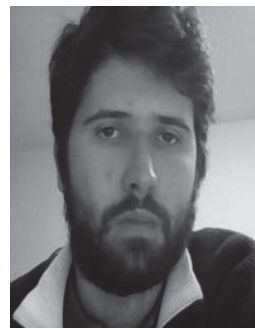

Broering, Elton Ferreira was born in Florianópolis, Santa Catarina, in 1994 $\mathrm{He}$ is currently pursuing the bachelor's degree in Telecommunications Engineering at the Instituto Federal de Santa Catarina. 


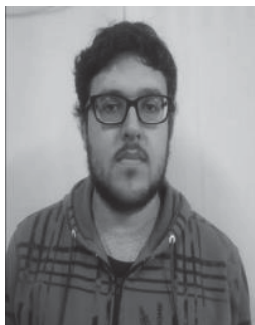

Jean Michel de Souza Sant'Ana was born in São José, Santa Catarina, Brazil. $\mathrm{He}$ is currently pursuing the bachelor's degree of Telecommunication Engeneering at Instituto Federal de Santa Catarina. He participated from 2014 to 2015 in the coding monitor program at Instituto Federal de Santa Catarina. Nowadays he is part of a research group about LoRa at Instituto Federal de Santa Catarina studying implementations and behaviors of this network.

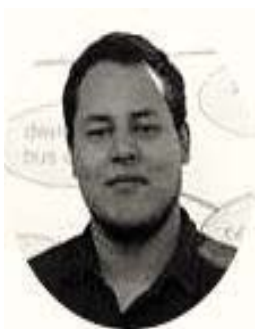

$\begin{array}{lll}\text { Thiago Bonotto } & \text { is a } \\ \text { Telecommunications } & \text { Engineering }\end{array}$ Student at Instituto Federal de Santa Catarina. He has developed research in areas like: software defined radio, computational vision and embedded system. Currently he is system analyst with experience in Linux environments. 